\title{
Clinical Study \\ Effects of Surgical and Dietary Weight Loss Therapy for Obesity on Gut Microbiota Composition and Nutrient Absorption
}

\author{
Antje Damms-Machado, ${ }^{1}$ Suparna Mitra, ${ }^{2}$ \\ Asja E. Schollenberger, ${ }^{1,3}$ Klaus Michael Kramer, ${ }^{4}$ Tobias Meile, ${ }^{3}$ \\ Alfred Königsrainer, ${ }^{3}$ Daniel H. Huson, ${ }^{2,5}$ and Stephan C. Bischoff ${ }^{1}$ \\ ${ }^{1}$ Department of Nutritional Medicine, University of Hohenheim, Fruwirthstraße 12, 70599 Stuttgart, Germany \\ ${ }^{2}$ Singapore Centre on Environmental Life Sciences Engineering, Nanyang Technological University, Singapore 637551 \\ ${ }^{3}$ Department of General, Visceral and Transplant Surgery, University of Tübingen, 72076 Tübingen, Germany \\ ${ }^{4}$ Department of General and Visceral Surgery, Chirurgische Klinik München-Bogenhausen, 81679 Munich, Germany \\ ${ }^{5}$ Center for Bioinformatics, University of Tübingen, 72076 Tübingen, Germany
}

Correspondence should be addressed to Stephan C. Bischoff; bischoff.stephan@uni-hohenheim.de

Received 27 August 2014; Accepted 10 October 2014

Academic Editor: Kuo-Sheng Hung

Copyright (c) 2015 Antje Damms-Machado et al. This is an open access article distributed under the Creative Commons Attribution License, which permits unrestricted use, distribution, and reproduction in any medium, provided the original work is properly cited.

Evidence suggests a correlation between the gut microbiota composition and weight loss caused by caloric restriction. Laparoscopic sleeve gastrectomy (LSG), a surgical intervention for obesity, is classified as predominantly restrictive procedure. In this study we investigated functional weight loss mechanisms with regard to gut microbial changes and energy harvest induced by LSG and a very low calorie diet in ten obese subjects ( $n=5$ per group) demonstrating identical weight loss during a follow-up period of six months. For gut microbiome analysis next generation sequencing was performed and faeces were analyzed for targeted metabolomics. The energy-reabsorbing potential of the gut microbiota decreased following LSG, indicated by the Bacteroidetes/Firmicutes ratio, but increased during diet. Changes in butyrate-producing bacterial species were responsible for the Firmicutes changes in both groups. No alteration of faecal butyrate was observed, but the microbial capacity for butyrate fermentation decreased following LSG and increased following dietetic intervention. LSG resulted in enhanced faecal excretion of nonesterified fatty acids and bile acids. LSG, but not dietetic restriction, improved the obesity-associated gut microbiota composition towards a lean microbiome phenotype. Moreover, LSG increased malabsorption due to loss in energy-rich faecal substrates and impairment of bile acid circulation. This trial is registered with ClinicalTrials.gov NCT01344525.

\section{Introduction}

Obesity is a worldwide health problem that reaches epidemic dimensions and is accompanied by a tremendously rising prevalence of related comorbidities, such as type 2 diabetes, hypertension, cardiovascular disease, nonalcoholic fatty liver disease, arthritis, depression, and certain cancers. In 2008, approximately 1.46 billion adults worldwide were overweight and 502 million adults were obese [1]. By now, mortality caused by overweight and obesity exceeds the number of deaths linked to underweight [2]. The success rates of suggested obesity prevention and treatment strategies including lifestyle modification, behavioural therapy, and pharmacotherapy are dissatisfactory and lack efficacy in the management of morbid obesity [3]. Surgical interventions are currently the most effective evidence-based approach towards clinically significant and sustainable weight loss along with reduction in mortality and obesity-related comorbidities $[4,5]$.

Bariatric operations experienced an exponential rise during the past few decades in matters of frequency, but also in the evolution of operation types and techniques [6-8]. Laparoscopic sleeve gastrectomy (LSG) is gaining popularity both as a single-staged and revisional operation and proved to be a simple and safe technique with promising short-term and midterm efficacy [9-11]. By partitioning and removal of 
a majority of the stomach parallel to downsizing the greater curvature while maintaining the gastrointestinal continuity, LSG was commonly classified as a solely restrictive procedure $[12,13]$. The latest evidence recognizes a more sophisticated mechanism of LSG involving alteration of gut hormones such as ghrelin, peptide YY, and glucagon-like peptide [14]. Moreover, recent studies demonstrate an accelerated gastric emptying and small bowel transit time possibly also responsible for the weight-reducing effect [15-19]. Thus, LSG seems to manifest itself as metabolic operation by supporting the "hindgut theory" suggesting that an expedite delivery of unabsorbed, incompletely digested nutrients to the distal intestine results in stimulation of hormonal changes leading to improved glycemic control [20]. However, the complex mechanisms resulting in the beneficial effects of LSG and the impact of surgically modified food ingestion and digestion remain poorly understood.

Besides diet, lifestyle, genetics, and environment, the gut microbiota has been recognized most recently as a potential contributor to the mechanisms of obesity and the metabolic syndrome. The nutrient load is a key variable that can influence the human gut microbiota, which in turn plays a role in nutrient absorption and the regulation of nutrient harvest [21]. One of the main bacterial phyla, the Firmicutes, more efficiently extracts calories from carbohydrates than Bacteroidetes by fermentation of otherwise indigestible food components to short-chain fatty acids (SCFA). Such metabolites are absorbed through the intestinal mucosa $[21,22]$, thus maximizing the energy supply provided by the diet.

The bariatric procedure Roux-en- $Y$ bypass has been shown to result in an increase in Firmicutes and decrease in Bacteroidetes in animal models [23] and humans [24, 25] and to reflect alterations due to the surgical induced anatomical changes in the gastrointestinal tract, while to the best of our knowledge no information is available on the impact of the increasingly popular, and, compared to gastric bypass, similarly effective bariatric sleeve gastrectomy.

In this context, we conducted a longitudinal pilot study over a period of six months including three time points, aiming to provide insight into the effect of LSG as well as a purely restrictive dietary approach for obesity therapy. We studied effects on gut microbiota, on the microbial metabolic capacity, as well as on faecal metabolic profiles using recent high-throughput "omics"-technologies. The solely restrictive and similar effective dietary therapy might enhance our understanding of other mechanisms than surgically induced food restriction that might be of importance for weight loss following LSG.

\section{Methods}

2.1. Weight Loss Interventions. Subject assignment to the dietary and surgical intervention was based on current evidence-based guidelines [26, 27].

2.1.1. Laparoscopic Sleeve Gastrectomy (LSG). LSG was performed at the University Hospital for General, Visceral and Transplant Surgery, Tübingen, Germany. Included patients met the criteria of the German national S3 guideline for the surgical treatment of morbid obesity [26]. The indication for LSG was approved by the local interdisciplinary case conference. LSG was performed by one surgeon (Klaus Michael Kramer) according to a standardized procedure as described previously [28]. Subjects undergoing LSG did not receive any kind of preoperative diet. Postoperative diet progression followed a gradual return to solid foods within a maximum of 8 weeks [29], with a caloric goal comparable to the group receiving the very low calorie diet $(\mathrm{VLCD})(=800 \mathrm{kcal} / \mathrm{d})$. A total energy intake around this magnitude three months after gastric resection is usually reported by our LSG patients to the dieticians guiding the post-operative nutrition care program. This is also confirmed by data indicating daily calorie intakes of $710 \mathrm{kcal}$ at three months [30] and $930 \pm 29 \mathrm{kcal} / \mathrm{d}$ at four months [31] postoperatively. Moreover, subjects were advised to follow a protein-rich, low-fat diet. In particular within the first three months the diet was very low in fiber, due to the postoperative nutrition care program ((1) liquids, (2) pureed, (3) soft solids, and (4) regular foods) as well as postoperative infeasibilities of fiber-rich, bulky foods.

2.1.2. Very Low Calorie Diet Program (VLCD). The multidisciplinary weight loss program (OPTIFAST 52) causes an average relative weight loss of $19.0 \%$ in men and $17.4 \%$ in women within one year [32]. Briefly, the program consists of a five-phase lifestyle modification over 52 weeks, including (i) a 1-week introduction during which a detailed nutrition analysis is performed; (ii) a 12 -week period of very low calorie diet (VLCD) (800 kcal/day) during which participants consume a formula diet exclusively (daily consumption of 5 sachets at $160 \mathrm{kcal}$ each, Optifast 800 formula, Nestlé Inc.); (iii) a 6-week refeeding phase; (iv) a 7-week stabilization phase; (v) a 26-week maintenance phase (the latter phase was not included for followup in this study). Besides medical examination, exercise units, and nutrition counselling all phases are accompanied by extensive behavioural modification lessons. More detailed information about the program is provided in a previous report [32].

2.2. Subject Selection and Biomaterial Sampling. Selected subjects were participants of a multicenter clinical trial, research project "Obesity and the Gastrointestinal Tract" within the Competence Network Obesity (registered with ClinicalTrials.gov NCT01344525), approved by the ethics committee of the University Hospital of Tübingen, Germany. Written informed consent was obtained from every subject prior to participation. Selection criteria included similar extent of weight loss, similar age, sex (only females were included), nondiabetics, and subject's affiliation to the same enterotype (Bacteroides-enterotype) to minimize interindividual variability. Faecal analysis was performed in 10 unrelated subjects with obesity grade III at three time points: one day before, as well as 3 and 6 months after LSG $(n=5)$ or the dietary weight loss regimen $(n=5)$, respectively. Short-chain fatty acids were analyzed in additional 10 subjects per group $(n=15$ LSG, $n=15$ VLCD), which met the same selection criteria. Results of gut microbiota analysis are shown for complete 
longitudinal datasets of 6 subjects ( $n=3$ LSG, $n=3$ VLCD). None of the subjects had a history of chronic gastrointestinal problems or current gastrointestinal symptoms and none had received antibiotic and pre- or probiotic agents within 3 months before sample collection. Of the same passage, native faecal specimens were collected in stool collection tubes for metabolite extraction and also in tubes containing DNA stabilizer (Stratec Molecular, Berlin, Germany) for isolation of microbial DNA. All samples were stored at $-80^{\circ} \mathrm{C}$ until analysis.

\subsection{Analysis of Gut Microbiota by SOLiD Long-Mate-Paired} Shotgun Sequencing. Faecal sample preparation and SOLiD (sequencing by oligonucleotide ligation and detection) shotgun sequencingwas performed as described previously [33]. Briefly, microbial DNA was isolated using the PSP Spin Stool DNA Plus Kit with lyses enhancer (Stratec Molecular, Berlin, Germany) as described by the manufacturer. Long-matepaired libraries with mate-paired distances of 300-900 bp apart from the whole microbial DNA (with a maximum at $800 \mathrm{bp}$ ) were generated by randomly shearing in a microTube format using the Covaris S2 sonicator (Covaris, Woburn, MA, USA), according to the mate-paired library construction protocol (Applied Biosystems, Foster City, CA, USA). The fragmentation protocol was mildly adapted to duty cycle $5 \%$, intensity 3 , cycles per burst 200 , at $4^{\circ} \mathrm{C}$. Fragmentation times were adjusted to $15 \mathrm{sec}$. No size selection was performed. Finished libraries were clonally amplified on paramagnetic beads, deposited onto a glass slide, and sequenced according to standard Applied Biosystems protocols using the SOLiD 4 System (Applied Biosystems, Foster City, CA, USA).

\subsection{Taxonomic, Functional, and Comparative Analysis of} Sequencing Data. The procedure for data processing was performed as previously reported [33]. In brief, the first step of data processing involved conversion of reads to the "fasta" format, while all the reads with less than 20 base pairs length or with base quality values below 18 were discarded. Reads of length $40 \mathrm{bp}$ or above from both forward and reverse files, together with their all mates $(>=20 \mathrm{bp})$, were considered. The adapters from both the files (" $\mathrm{T}$ " from the forward mates and " $G$ " from the reverse mates) were removed and all of these sequences were aligned against NCBI-NR database of nonredundant protein sequences [34] using BLASTX [35]. After performing the BLAST comparison, both output files were imported and analysed using the paired-end protocol of MEGAN 4 [36] (parameter settings min score $=35$, top percent $=10$, and min support $=25$ ) using adjustments according to Mitra et al. [33]. Finally, all the files were compared based on their taxonomic content.

2.5. Metabolite Extraction from Faecal Samples. Specimens were homogenized with a spatula and a predetermined mass (1 g) was placed into test tubes using a faecal sample preparation kit (Roche Diagnostics GmbH, Mannheim, Germany). We added $10 \mathrm{~mL}$ of extraction buffer $(\mathrm{EtOH}(>96 \%) / 10 \mathrm{mM}$ phosphate buffer ( $\mathrm{pH} 7,2), 85: 15(\mathrm{v} / \mathrm{v}))$ that had been cooled on dry ice to each sample $(1: 10[\mathrm{w} / \mathrm{v}])$ and homogenized it for 2 min using a Vortex mixer. Extracts were then vigorously shaken on ice for 2.5 hours with an orbital shaker. Subsequently, specimens were sonicated at $70 \mathrm{~W}$ for $1 \mathrm{~min}$ in an ice bath using an ultrasonic homogenizer (Sonopuls HD 2070, Bandelin GmbH \& Co. KG, Berlin, Germany). Next, faecal extracts were centrifuged $\left(800 \times \mathrm{g}, 0^{\circ} \mathrm{C}\right)$ for $10 \mathrm{~min}$. The supernatant was separated and again centrifuged $(20.800 \times \mathrm{g}$, $\left.5^{\circ} \mathrm{C}, 10 \mathrm{~min}\right)$. The obtained supernatant was stored at $-80^{\circ} \mathrm{C}$ until further analysis.

2.6. Mass Spectrometric Analysis of Faecal Fatty Acids and Bile Acids. A highly selective reversed phase LC-MS/MS analysis method performed in negative MRM detection mode was applied to determine the concentration of bile acids in faecal extracts. Samples were extracted via dried filter spot technique and measured by LC-ESI-MS/MS with an ABSciex 4000 QTrapTM tandem mass spectrometry instrument (AB Sciex, Darmstadt, Germany). Eicosanoids and other oxidized polyunsaturated fatty acids were determined by HPLCtandem mass spectrometry (LC-MS/MS) with multiple reaction monitoring (MRM) in negative mode using a SCIEX API 4000 QTrap mass spectrometer with electrospray ionization instrument (AB Sciex, Darmstadt, Germany) [37].

For determination of faecal SCFA human stool samples were freeze-dried and subsequently analyzed using gas chromatography. Briefly, the samples were weighted; then an extraction solution $\left(\mathrm{H}_{2} \mathrm{O}, \mathrm{HCL} 37 \%, 720 \mu \mathrm{L}\right.$ heptanoic acid) and glass beads ( $\varnothing 3 \mathrm{~mm})$ were added and the mixture was homogenized for $5 \mathrm{~min}$. After that it was left for $15 \mathrm{~min}$ and subsequently centrifuged for another $15 \mathrm{~min}(2.500 \times \mathrm{g}$ at $4^{\circ} \mathrm{C}$.) Concentrations of SCFA were determined in the supernatant, after adding trifluoroacetic acid, resting for $8 \mathrm{~h}$ and centrifugation for $90 \mathrm{~min}\left(2.500 \times \mathrm{g}\right.$ at $\left.4^{\circ} \mathrm{C}\right)$, and addition of internal standards using a Shimadzu GC 14A gas chromatograph (Shimadzu, Kyoto, Japan) equipped with a capillary column Roti Cap-FFAP $(30 \mathrm{~m} \times 0,32 \mathrm{~mm}$, df 0,5 $\mu \mathrm{m})$ (Carl Roth, Karlsruhe, Germany). Class-VP 4.2 software was used for data processing.

2.7. Multivariate Data Analysis and Statistics. Both taxonomically assigned sequencing reads and faecal metabolomics data were subjected to multivariate data analysis using the SIMCA-P+ 13.0 software (Umetric, Umea, Sweden). For the microbiome data, normalized Euclidean distances of the phylogenetic tree on each taxonomic level were calculated. For each dataset, a discriminant model was built with mean centred and unit variance scaled data using orthogonal partial least square discriminant analysis (OPLS-DA) to investigate differences between pre- and postintervention effects. In the model, the postintervention classification conjoined month 3 and month 6 data aiming to identify time-persistent markers. The quality and robustness of the models were evaluated by the cumulative $R^{2} Y$ and $Q^{2}$ values, where $R^{2} Y$ is a quantitative measure of the goodness of fit and $Q^{2}$ summarizes the model's goodness of prediction. 7-fold cross validation and permutation tests based on 200 permutations were performed to test for validity of the model and overfit of class assignment. Identification of prominent differences 
TABLE 1: Anthropometric and clinical parameters in the dietary (VLCD) and sleeve gastrectomy (LSG) intervention group before (month 0) intervention and after 3 and 6 months.

\begin{tabular}{|c|c|c|c|c|c|c|c|c|}
\hline & \multicolumn{4}{|c|}{ VLCD } & \multicolumn{4}{|c|}{ LSG } \\
\hline & Month 0 & Month 3 & Month 6 & $P^{*}$ trend & Month 0 & Month 3 & Month 6 & $P^{*}$ trend \\
\hline Weight $[\mathrm{kg}]$ & $121.1 \pm 4.9$ & $100.4 \pm 4.5$ & $91.3 \pm 3.9$ & $0.0014^{* *}$ & $127.5 \pm 1.2$ & $107.0 \pm 2.4$ & $97.1 \pm 3.0$ & $<0.0001^{* * *}$ \\
\hline BMI $\left[\mathrm{kg} / \mathrm{m}^{2}\right]$ & $40.2 \pm 1.0$ & $33.2 \pm 1.0$ & $30.3 \pm 0.6$ & $<0.0001^{* * *}$ & $45.8 \pm 0.9$ & $38.4 \pm 1.2$ & $35.0 \pm 1.3$ & $<0.0001^{* * *}$ \\
\hline Waist $[\mathrm{cm}]$ & $114.7 \pm 5.2$ & $104.8 \pm 1.9$ & $99.3 \pm 1.7$ & $0.0212^{*}$ & $138.3 \pm 3.6$ & $122.0 \pm 4.7$ & $112.0 \pm 4.2$ & $0.0027^{* *}$ \\
\hline Weight loss $[\mathrm{kg}]$ & & $20.7 \pm 0.4$ & $29.8 \pm 1.0$ & $<0.0001^{* * *}$ & & $20.5 \pm 1.2$ & $30.4 \pm 1.8$ & $0.0017^{* *}$ \\
\hline RWL [\%] & & $17.2 \pm 0.8$ & $24.6 \pm 0.8$ & $0.0002^{* * *}$ & & $16.1 \pm 1.1$ & $23.9 \pm 1.6$ & $0.0044^{* *}$ \\
\hline BP syst. [mmHG] & $120.0 \pm 4.5$ & $108.3 \pm 7.9$ & $108.3 \pm 1.3$ & 0.2352 & $143.3 \pm 13.7$ & $110.0 \pm 8.9$ & $106.7 \pm 2.6$ & $0.0351^{*}$ \\
\hline BP diast. $[\mathrm{mmHG}]$ & $83.3 \pm 1.3$ & $75.0 \pm 3.9$ & $73.3 \pm 1.3$ & $0.0321^{*}$ & $85.0 \pm 9.7$ & $66.7 \pm 2.6$ & $70.0 \pm 0.0$ & 0.1002 \\
\hline Glucose $[\mathrm{mg} / \mathrm{dL}]$ & $102.0 \pm 2.1$ & $96.0 \pm 0.8$ & $88.3 \pm 2.5$ & $0.0010^{* * *}$ & $125.3 \pm 10.4$ & $94.3 \pm 7.6$ & $105.0 \pm 3.8$ & $0.0436^{*}$ \\
\hline $\mathrm{HbAlC}[\%]$ & $5.6 \pm 0.1$ & $5.3 \pm 0.2$ & $5.3 \pm 0.0$ & 0.2186 & $5.5 \pm 0.1$ & $5.3 \pm 0.1$ & $5.3 \pm 0.2$ & 0.5705 \\
\hline Triglycerides [mg/dL] & $218.0 \pm 30.9$ & $113.7 \pm 6.1$ & $125.7 \pm 9.1$ & $0.0039^{* *}$ & $169.3 \pm 17.4$ & $119.0 \pm 7.5$ & $117.0 \pm 1.6$ & $0.0086^{* *}$ \\
\hline Cholesterol [mg/dL] & $224.7 \pm 15.9$ & $164.0 \pm 2.4$ & $176.3 \pm 16.3$ & $0.0165^{*}$ & $206.0 \pm 17.5$ & $188.3 \pm 16.1$ & $218.7 \pm 16.9$ & 0.4640 \\
\hline $\mathrm{HDL}[\mathrm{mg} / \mathrm{dL}]$ & $51.3 \pm 2.7$ & $42.3 \pm 1.8$ & $54.3 \pm 2.9$ & $0.0147^{*}$ & $52.0 \pm 3.9$ & $44.7 \pm 4.2$ & $48.3 \pm 4.3$ & 0.4827 \\
\hline $\mathrm{LDL}[\mathrm{mg} / \mathrm{dL}]$ & $143.3 \pm 12.8$ & $104.3 \pm 4.5$ & $108.7 \pm 11.5$ & $0.0383^{*}$ & $133.0 \pm 21.2$ & $118.3 \pm 21.4$ & $143.0 \pm 20.8$ & 0.7147 \\
\hline
\end{tabular}

${ }^{*}$ Within group comparison.

between pre- and postintervention was performed according to the method suggested by Rantalainen et al. [38]. In brief, the predictive regression coefficients $(P$ (corr)) from each model were used to identify the most prominently changing variables, considering a $P($ corr $)>0.75$ and $P$ (corr $)<-0.75$, respectively, as cutoff for significance $(*)$. More detailed analysis of parameters selected by multivariate analysis was performed using conventional statistical tests. Accordingly paired $t$-tests or Wilcoxon matched pair tests were used for comparison of two time points and repeated measures analysis of variance (one-way-ANOVA with Tukey post hoc testing) or Friedmann test for time courses were conducted after testing for Gaussian distribution using D'AgostinoPearson tests. Absolute and percentage values are presented as mean \pm SEM.

\section{Results}

3.1. Weight Loss and Clinical Parameters. The 10 obese individuals were all female, age $48 \pm 3$ years, and nonsmokers. Baseline anthropometric measures weight, BMI, and waist circumference did not significantly differ between both intervention groups. Bariatric sleeve operation resulted in a relative weight loss (RWL) of $16.1 \pm 1.1 \%$ after 3 months and $23.9 \pm 1.6 \%$ at months 6 . There were no significantly different weight loss characteristics (absolute and relative weight loss) after dietary intervention, which resulted in a RWL of $17.2 \pm 0.8 \%$ at month 3 and $24.6 \pm 0.8 \%$ after 6 months of intervention. The detailed pre- and postoperative anthropometric and clinical parameters are given in Table 1.

3.2. Gut Microbiota Composition. Analysis of similarities between subjects and time points based on calculation of normalized Euclidean distances revealed profound changes of faecal microbial community composition after LSG, whereas the VLCD intervention resulted in a more varied picture with less distinct dissimilarity between time points (see SI-Figure 1 in Supplementary Material available online at http://dx.doi.org/10.1155/2014/806248). Detailed post-interventional alterations of bacterial groups on all taxonomic levels were identified using coefficient plots of OPLS-DA models (SI-Figure 2 and SI-Table 1). Both interventions resulted in changes of the Bacteroidetes : Firmicutes ratio, but with an inverse relationship between the main phyla (Figure 1). While LSG resulted in a distinct increase in Bacteroidetes $(P($ corr $)=0.85)$ and a decline in the abundance of Firmicutes $(P$ (corr) $=-0.85)$, the dietary intervention resulted in reduced Bacteroidetes in favour of Firmicutes. The Bacteroidetes/Firmicutes ratio changed from $14.2 \pm 7.9$ to $4.8 \pm 0.5$ (month 3 ) and $3.7 \pm 1.4$ (month 6) following dietary intervention, whereas in the LSG group, this ratio increased from $5.9 \pm 2.1$ to $10.4 \pm 1.4$ (month 3 ) and $13.8 \pm 3.0$ (month 6 ). In the LSG group, the number of Bacteroidetes showed a negative correlation with body weight $(r=-0.61, P=$ $0.05)$ while Firmicutes numbers were positively correlated with body weight $(r=0.65, P=0.05)$. In contrast, in the dietary group body weight correlated significantly positive with Bacteroidetes $(r=0.67, P=0.05)$ and inversely with Firmicutes $(r=-0.68, P<0.05)$. A figure of the abundance of bacterial groups at the class level for each subject and timepoint is presented in SI-Figure 3.

In each group 9 bacterial species of the gut microbiota were identified, which consistently altered pre- to postintervention. Figure 2 depicts changes on species level for both groups. Apart from two species of the Bacteroidetes genus in the LSG group (Bacteroides sp. 3_1_40A $P$ (corr) $=-0.92$ and Bacteroides vulgatus $P($ corr $)=-0.78$ ), all alterations occurred in species assigned to Firmicutes phylum. Except for a postoperative increase in Faecalibacterium prausnitzii $(P($ corr $)=0.81)$, LSG resulted in a decrease of several Firmicutes species. In the latter group all Bacteroidetes 

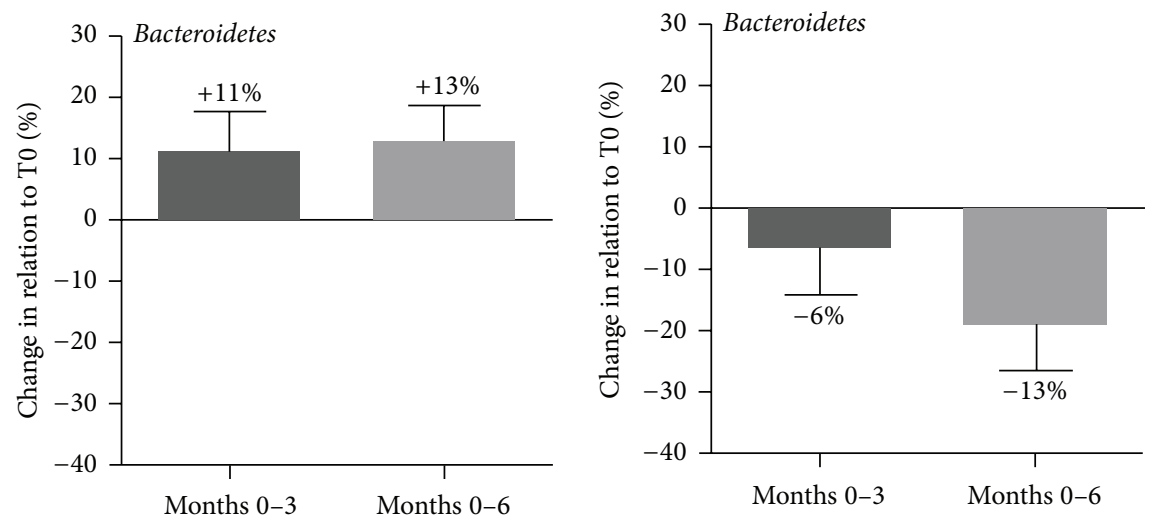

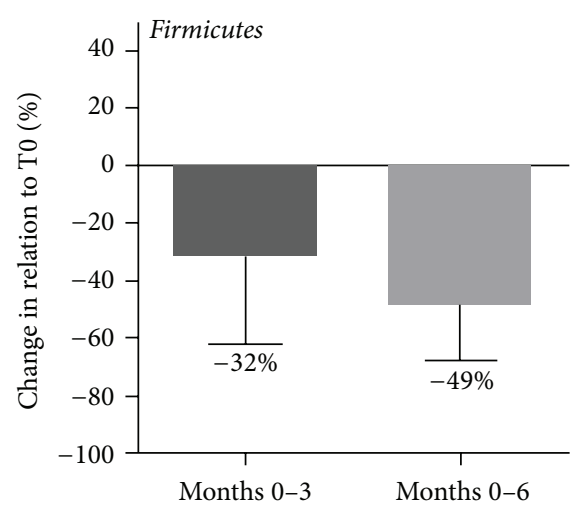

(a) LSG

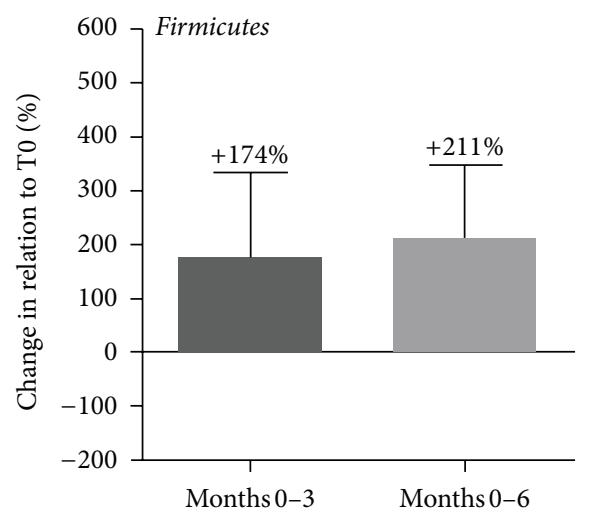

(b) VLCD

FIGURE 1: Alterations in the main phyla Bacteroidetes and Firmicutes of the human intestinal microbiota after three and six months of weight loss therapy for morbid obesity: (a) laparoscopic sleeve gastrectomy (LSG) and (b) very low calorie diet (VLCD).

subgroups increased $(P($ corr $)=0.87)$. The postoperative reduction in Firmicutes following LSG was caused by a decline of bacterial subgroups belonging to Clostridial clusters IV and XIV, which include species of Clostridium, Eubacterium, Faecalibacterium, Dorea, and Coprococcus. In particular known butyrate-producing bacteria (Coprococcus sp. $P$ (corr) $=-0.88$, Eubacterium rectale $P($ corr $)=-0.79$, Ruminococcus obeum $P$ (corr) $=-0.82$, and Lachnospiraceae bacterium 5_1_63FFA $P$ (corr) $=-0.85$ ) experienced a decline in the postoperative period. In contrast, we observed a rise in specific Firmicutes species in the course of the VLCD program. These changes also pertained to butyrate-producing bacterial strains (Butyrivibrio fibrisolvens $P$ (corr) $=0.80$, Clostridium saccharolyticum $(P($ corr $)=0.94)$, Eubacterium limosum $(P($ corr $)=0.90)$, and Blautia hydrogenotrophica $(P($ corr $)=0.88))$, but here resulting in an increase in those microbes.

\subsection{Microbial Metabolic Capacity for Butyrate Fermentation} Pathway. Based on the findings of predominantly alterations in butyrate-synthesizing bacteria, we analyzed the microbial metabolic capacity of the gut microbiota for the main KEGG orthologues (acetyl-CoA-acetyl transferase (K00074), butyrate kinase (K00929), and beta-hydroxy-butyryl-CoA dehydrogenase (K00626)) involved in the fermentation pathway to butyrate. Summed values tended to result in a mean increase of $380.2 \pm 52.5 \%$ in the metabolic capacity of these KEGG orthologues for butyrate fermentation after 6 months of VLCD program (K00074 227.3 $\pm 254.2 \%$, K00929 $102.3 \pm$ $61.5 \%$, and K00626 50.5 $\pm 27.45 \%)$ and a decrease for the LSG intervention $-184.4 \pm 63.0 \%$ (K00074-126.7 $489.7 \%$, K00929 $64.4 \pm 118.0 \%$, and $\mathrm{K} 00626-122.2 \pm 91.0 \%)$, respectively (Figure 3).

3.4. Faecal Fatty Acid Excretion and Bile Acid Metabolism. Both weight loss interventions, the VLCD regimen and LSG, did not reveal significant differences in faecal butyrate content and other SCFA, possibly because of low prebiotic substrate levels in the diet. Data on faecal SCFA concentrations were also performed in 10 additional and similarly matched subjects per intervention group and confirmed unaltered SCFA concentrations after both weight loss strategies (Table 2).

Analysis of faecal excretion of a total of 51 middle- and long-chain nonesterified fatty acids (NEFAs) revealed a trend to a decline in faecal concentrations of 31 NEFAs 6 months after the VLCD program (ns $(P=0.2283)$ for summed values); contrariwise, there was an increased loss of overall 

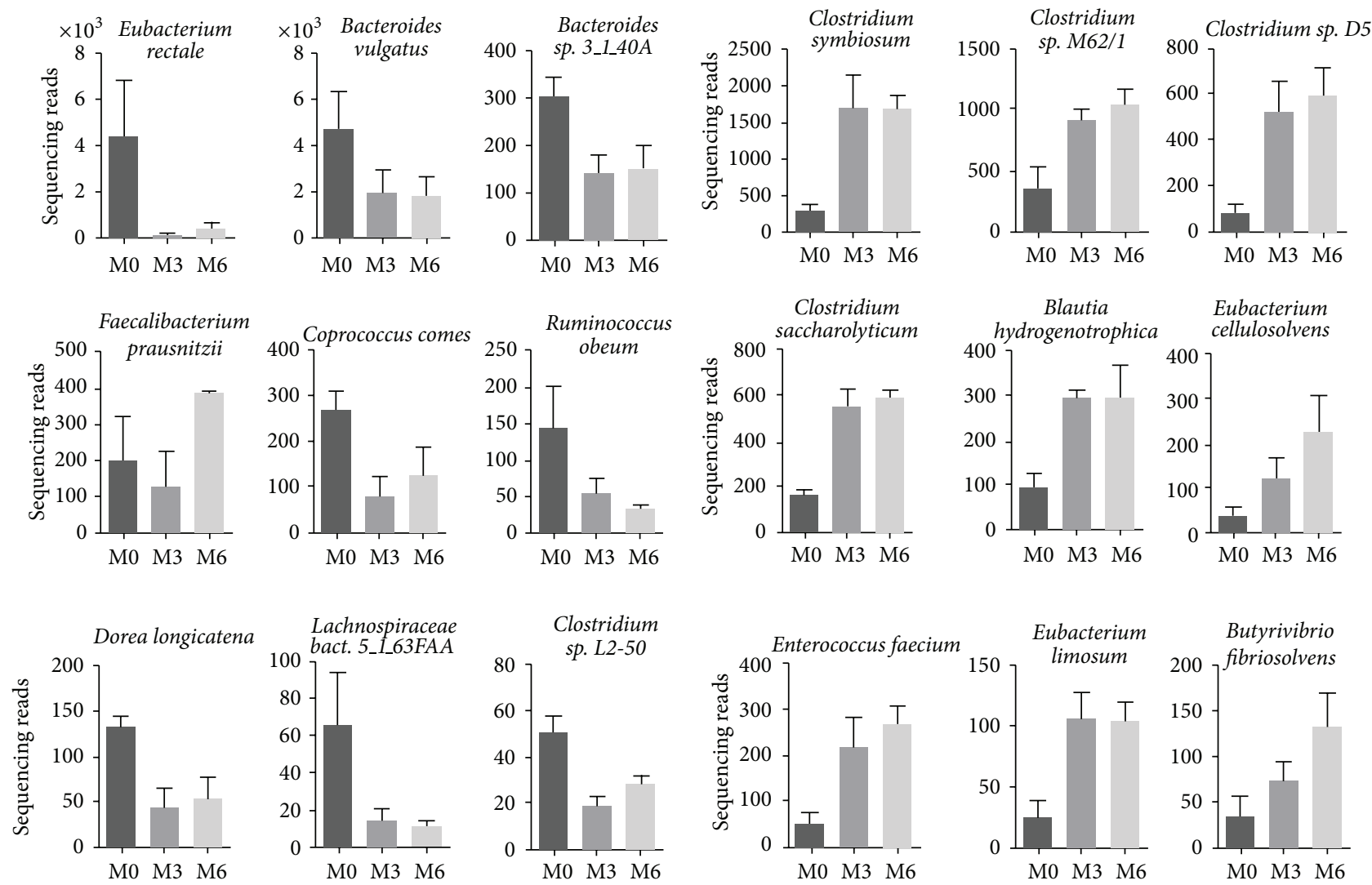

(a) LSG

(b) VLCD

Figure 2: Human gut microbiota changes on the taxonomic species level induced by weight loss therapy for morbid obesity: (a) laparoscopic sleeve gastrectomy (LSG) and (b) very low calorie diet (VLCD). Shown are all bacterial species for which changes were significantly defined as a $P$ (corr) $>0.75$ or $<-0.75$ derived from OPLS-DA models.

TABLE 2: Fecal short-chain fatty acid concentrations (mmol/100 g dry weight) in the dietary (VLCD; $n=15)$ and sleeve gastrectomy (LSG, $n=15$ ) intervention groups before intervention (month 0 ) and after 3 and 6 months.

\begin{tabular}{|c|c|c|c|c|c|c|c|c|}
\hline & \multicolumn{4}{|c|}{ VLCD } & \multicolumn{4}{|c|}{ LSG } \\
\hline & Month 0 & Month 3 & Month 6 & $P$ trend & Month 0 & Month 3 & Month 6 & $P$ trend \\
\hline Acetate & $20.3 \pm 3.8$ & $15.6 \pm 2.5$ & $29.6 \pm 1.9$ & 0.4041 & $10.3 \pm 1.7$ & $16.5 \pm 4.3$ & $14.2 \pm 3.0$ & 0.3909 \\
\hline Propionate & $5.8 \pm 1.2$ & $3.5 \pm 0.5$ & $3.7 \pm 0.5$ & 0.0954 & $3.5 \pm 0.7$ & $3.7 \pm 0.8$ & $3.5 \pm 0.7$ & 0.9743 \\
\hline n-Butyrate & $4.5 \pm 1.0$ & $2.5 \pm 0.6$ & $2.5 \pm 0.5$ & 0.1095 & $2.2 \pm 0.4$ & $2.2 \pm 0.6$ & $2.5 \pm 1.5$ & 0.9232 \\
\hline i-Butyrate & $0.6 \pm 0.1$ & $0.5 \pm 0.1$ & $0.5 \pm 0.1$ & 0.3393 & $0.4 \pm 0.1$ & $0.4 \pm 0.1$ & $1.0 \pm 0.4$ & 0.1973 \\
\hline i-Valeriate & $0.8 \pm 0.1$ & $0.7 \pm 0.1$ & $0.7 \pm 0.1$ & 0.8427 & $0.6 \pm 0.1$ & $0.6 \pm 0.1$ & $0.6 \pm 0.1$ & 0.8458 \\
\hline Caproate & $0.2 \pm 0.0$ & $0.2 \pm 0.0$ & $0.2 \pm 0.0$ & 0.6400 & $0.2 \pm 0.0$ & $0.2 \pm 0.0$ & $0.2 \pm 0.0$ & 0.8547 \\
\hline Total SCFA & $30.7 \pm 5.9$ & $22.4 \pm 3.8$ & $21.9 \pm 2.7$ & 0.2862 & $18.2 \pm 2.6$ & $23.7 \pm 6.3$ & $24.9 \pm 5.3$ & 0.6192 \\
\hline
\end{tabular}

SCFA: short chain fatty acids.

40 different fatty acids 6 months after LSG compared to preoperative values resulting in a faecal excretion of summed fatty acid concentrations from preoperative $1,726 \pm 1,093 \mu \mathrm{M}$ to $3,554 \pm 1,872 \mu \mathrm{M}$ at month 3 and $6,106 \pm 1,005 \mu \mathrm{M}$ at month $6(P=0.005)$ (Figure 4$)$.

Faecal metabolomics analysis of bile acids after LSG indicates postoperative increases in conjugated bile acids, while on contrary secondary bile acids (lithocholic acid, LCA; deoxycholic acid, DCA) slightly tended to decrease
(Figure 5), while there was no change in the VLCD group (data not shown). The conjugated bile acids, glycodeoxycholic acid (GDCA, $P($ corr $)=0.76)$, taurodeoxycholic acid (TDCA, $P($ corr $)=0.93)$, and taurochenodeoxycholic acid (TCDCA, $P($ corr $)=0.84)$, most prominently contributed to separation of pre- to postoperative profiles. This was also linked to a certain decrease of the microbial capacity for primary and secondary bile acid biosynthesis, which was averagely reduced by $-23.8 \pm 33.3 \%$ at month 3 and 

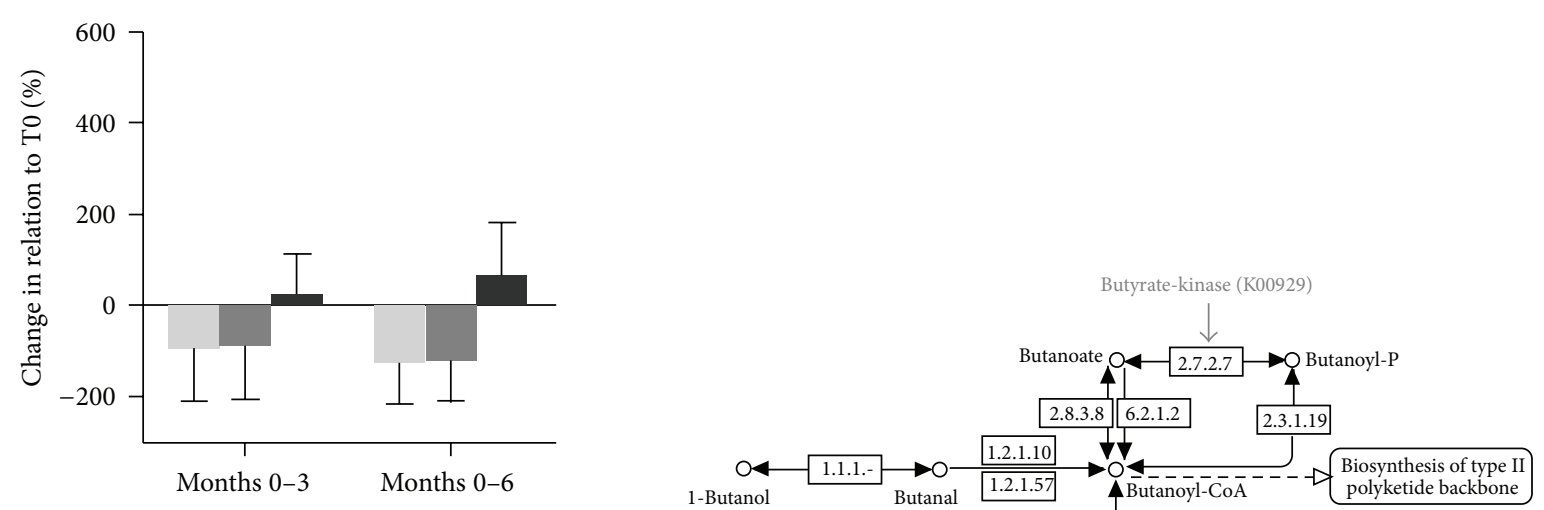

(a) LSG
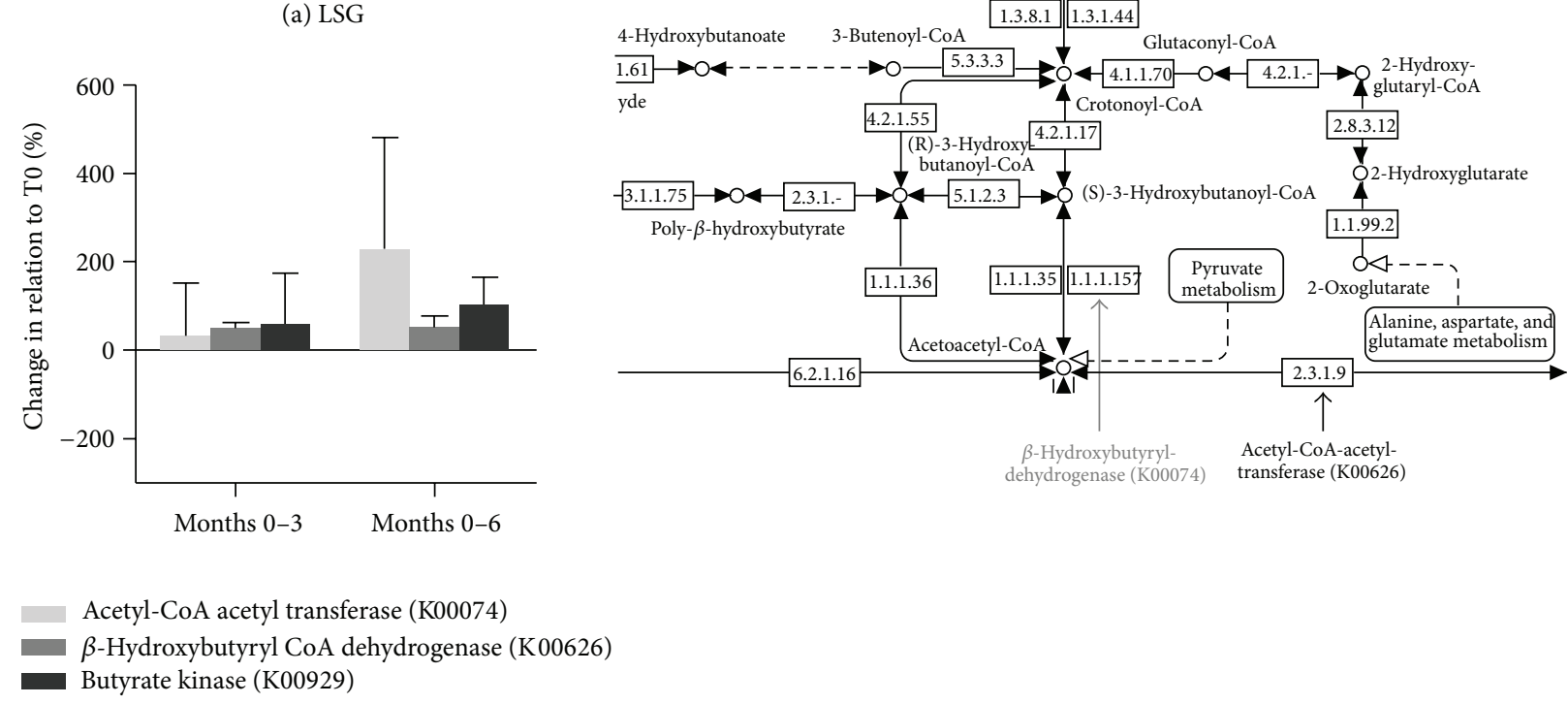

(b) VLCD

FIGURE 3: KEGG orthologues representing the microbial metabolic capacity of the human gut microbiota for butyrate fermentation after weight loss therapy for morbid obesity: (a) laparoscopic sleeve gastrectomy (LSG) and (b) very low calorie diet (VLCD). Shown are three KEGG orthologues (K00074, K00929, and K00626) with key functions in the fermentation pathway denoted in the KEGG map extract on the right bottom corner.

$-34.2 \pm 23.9 \%$ at month 6 compared to pre-LSG (SI-Figure 4 ). Correlation analysis of gut microbial changes observed after LSG also showed positive associations between faecal secondary bile acid concentrations and postoperatively reduced microbial species (correlation of LCA with Faecalibacterium prausnitzii: $r=0.68, P=0.04$; DCA with Clostridium sp. L250: $r=0.73, P=0.03$; Coprococcus comes: $r=0.63, P=0.04$; and Lachnospiraceae bacterium: $r=0.81, P=0.01$, resp.), though conjugated bile acids indicated negative correlation coefficients (SI-Figure 5).

\section{Discussion}

In this study we analyzed longitudinal data in the course of two different approaches of predominantly restrictive weight loss therapies, the bariatric procedure LSG, and a standardized calorie restricted formula diet.

The bariatric procedure LSG was followed by a clear reduction of the Firmicutes/Bacteroidetes ratio. The change in main phyla was also observed in studies, in which subjects reduced body weight with caloric restriction and physical activity [39], fat- or carbohydrate-reduced diets [40], and Roux-en-Y gastric bypass (RYGB) [25]. Thus, one driving factor of the altered faecal microbiota composition might be the bacterial adaptation to caloric restriction. Due to the associated fermentation activity, occurrence of lower levels of Firmicutes following LSG could result in a reduction of energy harvest besides caloric restriction and thus might benefit sustained weight loss and maintenance. However, this was not clearly confirmed by our data on a functional level as shown by the unaltered faecal SCFA concentrations. Possibly, the expected decrease in SCFA production would become visible only if subjects would be challenged at the time of faecal analysis to substantial amounts of prebiotics and fibres, the substrates needed by the bacteria to generate SCFA. On the other hand, we observed a decline in butyrate-producing bacterial species after the surgical procedure. These modifications indeed point to a postoperatively reduced dietary intake of complex carbohydrates with prebiotic properties, 


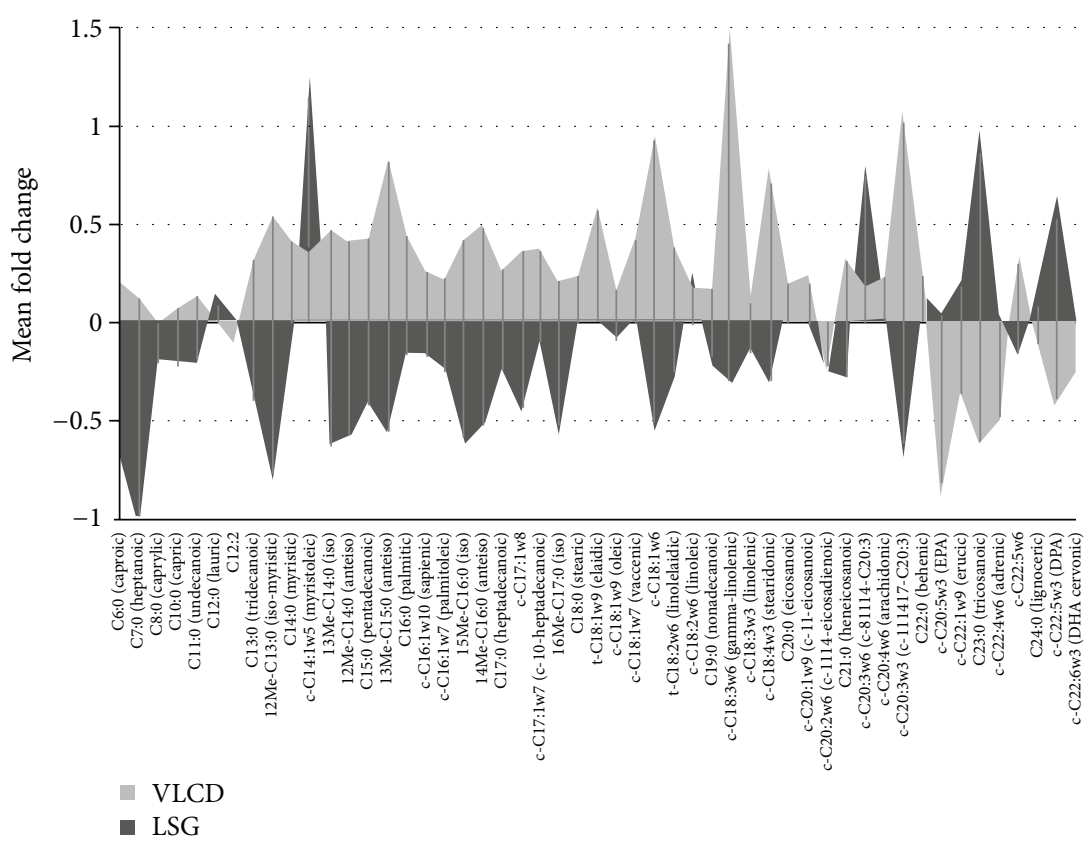

(a)

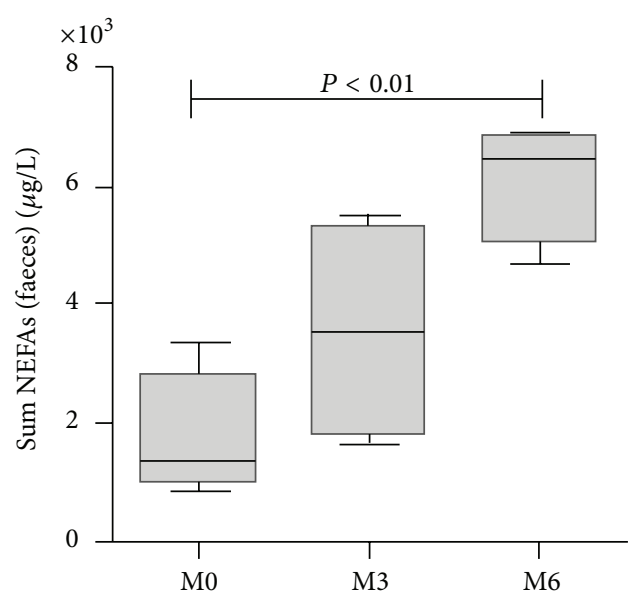

(b)

FIGURE 4: Faecal excretion of middle- and long chain nonesterified fatty acids (NEFAs): (a) mean fold changes of NEFA excretion 6 months after weight loss therapy for morbid obesity (light grey: laparoscopic sleeve gastrectomy (LSG) and dark grey: very low calorie diet (VLCD)). (b) Summed faecal concentrations of NEFAs in the course after laparoscopic sleeve gastrectomy (LSG).

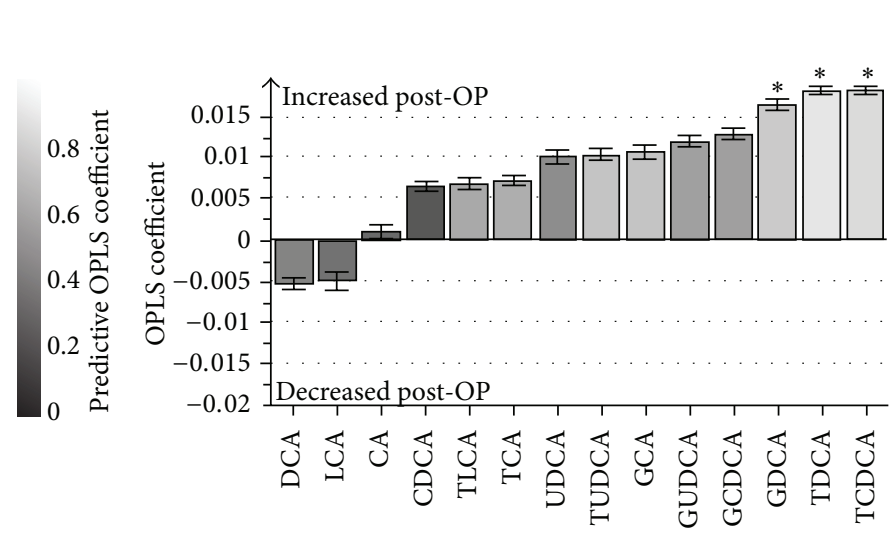

(a)

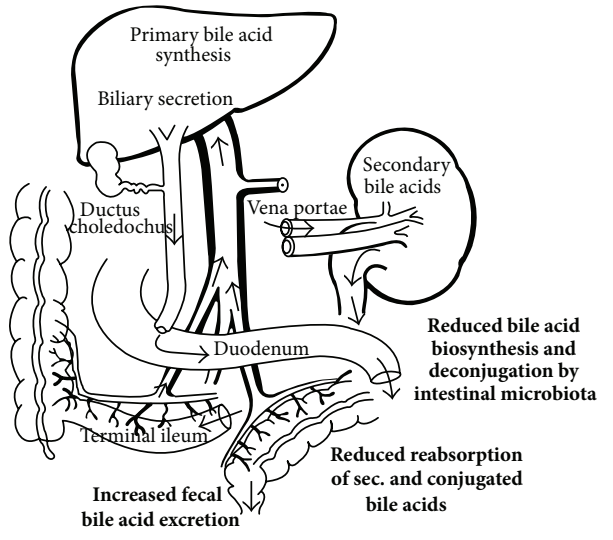

(b)

FIGURE 5: Faecal bile acids alterations six months after laparoscopic sleeve gastrectomy (LSG). OPLS-DA coefficient plot showing the increase or decrease in concentration for each of the bile acids identified by targeted profiling. The model compares 6 months post-LSG with preoperative values. The coefficient along the $y$-axis is a measure of both the magnitude and direction of change of bile acid concentrations. * indicates which metabolites contribute significantly to class discrimination (pre- to post-LSG; $P$ (corr) $>0.75$ and $P$ (corr) $<-0.75$ ) corresponding to the color scale code shown on the left hand. Error bars derived from calculation of Jack-knifing uncertainty measures. (b) Suggested impact of LSG on extrahepatic bile acid circulation based on the presented data.

especially resistant starch [22], which is consistent with the nutrition care program with slow progression from liquids to regular foods [29] as well as postoperative infeasibilities of fiber-rich, bulky foods after stomach resection. This change in diet after LSG can be explained by the anatomically induced food restriction, as well as a suggested more pronounced feeling of satiety induced by hormonal modulation [41]. Hence, in our subjects the observed modulation of the intestinal microbiota following LSG operation could be attributed to dietary influences, like reduced caloric restriction and fiber consumption.

Our data illustrate the less "invasive" impact of LSG, not only regarding anatomy but also regarding the gut microbiota, compared to RYGB. The latter procedure has 
been shown to lead to profound alterations of the intestinal microbiota [23-25]. Changes after RYGB can be explained to a large extent by an increased acid exposure to the gastric remnant and shortened small intestine as well as input of dissolved oxygen in the small bowel favouring growths of facultative anaerobe bacteria [24]. Moreover, the typical small intestinal microbiota might be relocated to the large intestine due to the more rapid flux of incompletely digested nutrients into the proximal gut [24]. These observations may contribute to creation of a cytotoxic environment in the bowel, [42] also leading to shifts in the gut microbial-host metabolic crosstalk as demonstrated by faecal, urinary, and host metabolomic profiles $[23,42,43]$. Thus, by maintaining the gastrointestinal continuity, LSG causes only moderate microbiota alterations with less severe negative consequences. Moreover, we also observed beneficial microbial changes during weight loss induced by LSG. Eubacterium rectale, which decreased after LSG operation, has previously been shown to positively correlate with obesity-related comorbidities [44], thus pointing to an influence of this microbe on host metabolic status. Furthermore, the study of Furet et al. [25], suggests that Faecalibacterium prausnitzii plays a role in low-grade inflammation pathologies such as obesity and diabetes and reports a reduction of this bacterial species after RYGB. Here we present that also LSG resulted in a decrease in Faecalibacterium prausnitzii numbers in obese subjects with preoperative impaired glucose tolerance.

In contrast, a dietary intervention with VLCD followed by a progressive adaptation to a low-fat, high-fiber, and energy-restricted diet resulted in a reduction of the Bacteroidetes/Firmicutes ratio along with weight loss. Uniformly, increased bacterial strains exclusively appertained to Firmicutes. Particularly, Butyrivibrio fibrisolvens, Clostridium saccharolyticum, Eubacterium limosum, and Blautia hydrogenotrophica represent bacteria, whose capability is the synthesis of the SCFA butyrate through fermentation of nondigestible nutrients in the upper GI tract. Moreover, growth of Coprobacillus and Holdemania, as well as Eubacterium cellulosolvens and Clostridium saccharolyticum, is favoured by an increase in fiber intake. Reasons for the modulation of microbiota composition might be the initial supply of the soluble fiber inulin included in the formula diet and the subsequent increased intake in dietary fiber, which was introduced by intensive nutritional training. Acetate and propionate, which are absorbed and transported to liver and other peripheral organs serving as substrates for gluconeogenesis and lipogenesis, represent an additional energy source for the host. In contrast, butyrate is primarily an energy substrate for colonic epithelial cells with positive properties in regard to integrity of the intestinal barrier [45]. Thus, despite an overall increase in Firmicutes at the phylum level following VLCD, which is generally associated with increased energy harvest for the host, the specific growth of the Firmicutes-related butyrate-producing species may result in positive metabolic effects. Nondigestible carbohydrates/prebiotics have been shown to reduce food intake by modulating the production of gastrointestinal peptides [46, 47]. Moreover, studies in murine models showed that activation of intestinal GLP1 cells improves glycemic and insulin response [47]. This is accompanied by an improved expression and activity of proteins, which are involved in the intestinal barrier function and additionally reinforced by normalization of the intestinal endocannabinoid system response (reviewed in [48]). The increase in beneficial butyrate-producing microbes induced by soluble fiber supplementation might propose a possibility to countervail the observed postoperative declines in these microbial species following LSG.

The functional analysis of the metabolic capacity suggests an increased expression of enzymes, which are involved in butyrate fermentation, and thus an increased enzymatic potential for fermentation of acetyl CoA to butyrate after the VLCD program and vice versa for LSG. However, the observed gut microbial alterations in both intervention groups did not support any consequences for energy harvest as indicated by unaltered faecal SCFAs. This may be again due to a too limited substrate supply, such as fiber and resistant starch during both restrictive therapies to provoke changes on a functional level.

Favorable changes in the blood lipid profile with dietary restriction and weight loss were only induced by VLCD. The postoperative increases of total cholesterol and LDL 6 months after LSG may be explained by consumption of foods with unfavorable fatty acid patterns, especially after adaptation to the resected stomach ( $>$ three months postoperatively), and due to the lack of a guided nutrition counseling/lifestyle program for these subjects, as it was performed in the VLCD group. The guided group-based physical exercise classes in the VLCD group may also have induced the increase in HDL concentrations, which were not observed in the LSG patients.

Interestingly, we found increased faecal concentrations of middle- and long-chain fatty acids following LSG, whereas VLCD intervention resulted in lower concentrations compared to baseline. Dietary fat intake usually correlates with faecal fat excretion [49], which was in line with a decline in faecal fatty acid loss in the VLCD group after a period of food restriction, especially a reduced dietary fat consumption. The reciprocal finding after LSG points to a postoperative state of (moderate) malabsorption with an augmented faecal excretion of fatty acids. In recent years, evidence was raising that resection of the stomach leads to an accelerated gastric emptying by increased peristalsis [15-18]. Thus, the resulting delivery of unabsorbed, incompletely digested nutrients to distal intestinal areas might result in an increased loss of energy via faeces. Augmented faecal fat excretion is also observed after RYGB $[50,51]$, pointing to equal mechanisms of LSG and RYGB with regard to some intestinal malabsorption as a mechanism for weight loss that can be explained by accelerated gastric emptying and small bowel transit time. This subject should be addressed in further investigations analyzing total caloric contents in faeces before and after LSG to evaluate its impact on caloric load and absorption.

The faecal bile acids data further indicate a surgically induced alteration in the enterohepatic circulation. This is in line with the observation of increased faecal excretion of middle- and long-chain fatty acids, as bile acids play an important role in fat digestion and absorption. The primary bile acids undergo bacterial degradation in the intestine, whereat the secondary bile acids DCA, LCA, and 
their oxidation products are produced. The data suggest a reduced metabolic capacity by the postoperative intestinal microbiota for this process. Salts of conjugated bile acids are effective solvents, which provide absorption of lipids in the intestine. These lipids are almost completely absorbed and revert to liver and gall bladder via enterohepatic circulation. Besides a possible influence of gut microbial alterations, the fact that the faecal excretion of conjugated bile acids increased postoperatively may also be explained by a faster gastrointestinal passage possibly leading to a certain level of bile acid malabsorption.

A limitation of the study is the fact that the postoperative diet was not exactly matched to the VLCD intervention, which impedes a reliable analysis to dissect whether the observed changes were due to the LSG anatomical changes or to dietary restriction after surgery. Due to the small number of patients subjected to the sequencing analysis of the intestinal microbiome, this study has to be recognized as a pilot study, which needs to be confirmed in further studies. However, we focused on a time series in well-matched subjects for identification of time-persistent alterations using information from detailed deep sequencing techniques. In contrast to Zhang et al. [24], who compared three obese patients who underwent bariatric gastric bypass operation with three unrelated obese subjects without intervention, the approach presented here aimed at identification of intraindividual intervention-induced changes. Moreover, a strength of our study is that, in contrast to other published studies analysing gut microbiota composition in obesity, our focus was not only on a description of a shift of entire bacterial communities like the main phyla but also on detailed analysis of gut microbiota changes down to the species level.

\section{Conclusions}

By preserving the gastrointestinal continuity LSG resulted only in moderate alterations of the intestinal microbiota. The modulations can be explained by weight loss and dietary food restriction, most likely by reduced fiber consumption, as shown by the increase in Bacteroidetes/Firmicutes ratio and decline in butyrate-producing bacterial strains. In contrast, VLCD leads to an increase in butyrate-producing bacteria, which can be explained by soluble fiber supply, pointing to a possibility for supplementation with favourable effects on gut microbiota also in the postoperative period after LSG. Moreover, our data indicate a state of moderate malabsorption with loss of energy-rich faecal substrates and altered bile acid metabolism induced by LSG, which question its classification as solely restrictive procedure.

\section{Conflict of Interests}

The authors declare that there is no conflict of interests regarding the publication of this paper.

\section{Acknowledgments}

The authors acknowledge the support of CeGaT (Center for Genomics and Transriptomics) GmbH, Tübingen, Germany, for performing sequencing analysis; Biocrates Life Sciences AG, Innsbruck, Austria; and Babende Institute, Würzburg, Germany, for technical analysis of faecal metabolites. The authors also thank Karin Förster-Fromme for methodological input and technical assistance. This work was supported by the Kompetenznetz Adipositas (Competence Network of Obesity) funded by the Federal Ministry of Education and Research (FKZ: 01GI0843).

\section{References}

[1] B. A. Swinburn, G. Sacks, K. D. Hall et al., "The global obesity pandemic: shaped by global drivers and local environments," The Lancet, vol. 378, no. 9793, pp. 804-814, 2011.

[2] WHO, "Obesity and overweight," Fact Sheet 311, World Health Organization, Geneva, Switzerland, 2012.

[3] J. G. Kral, R. A. Kava, P. M. Catalano, and B. J. Moore, "Severe obesity: the neglected epidemic," Obesity Facts, vol. 5, no. 2, pp. 254-269, 2012.

[4] L. Sjöström, M. Peltonen, P. Jacobson et al., "Bariatric surgery and long-term cardiovascular events," Journal of the American Medical Association, vol. 307, no. 1, pp. 56-65, 2012.

[5] M. Mitka, "Bariatric surgery continues to show benefits for patients with diabetes," Journal of the American Medical Association, vol. 307, no. 18, pp. 1901-1902, 2012.

[6] H. Buchwald and D. M. Oien, "Metabolic/bariatric surgery worldwide 2008," Obesity Surgery, vol. 19, no. 12, pp. 1605-1611, 2009.

[7] H. Buchwald and J. N. Buchwald, "Evolution of operative procedures for the management of morbid obesity 1950-2000," Obesity Surgery, vol. 12, no. 5, pp. 705-717, 2002.

[8] H. Buchwald and D. M. Oien, "Metabolic/bariatric surgery worldwide 2011," Obesity Surgery, vol. 23, no. 4, pp. 427-436, 2013.

[9] W. Abu-Jaish and R. J. Rosenthal, "Sleeve gastrectomy: a new surgical approach for morbid obesity," Expert Review of Gastroenterology and Hepatology, vol. 4, no. 1, pp. 101-119, 2010.

[10] A. P. Chambers, L. Jessen, K. K. Ryan et al., "Weightindependent changes in blood glucose homeostasis after gastric bypass or vertical sleeve gastrectomy in rats," Gastroenterology, vol. 141, no. 3, pp. 950-958, 2011.

[11] R. Peterli, B. Wölnerhanssen, T. Peters et al., "Improvement in glucose metabolism after bariatric surgery: comparison of laparoscopic roux-en-Y gastric bypass and laparoscopic sleeve gastrectomy: a prospective randomized trial," Annals of Surgery, vol. 250, no. 2, pp. 234-241, 2009.

[12] X. Shi, S. Karmali, A. M. Sharma, and D. W. Birch, "A review of Laparoscopic sleeve gastrectomy for morbid obesity," Obesity Surgery, vol. 20, no. 8, pp. 1171-1177, 2010.

[13] A. A. Gumbs, M. Gagner, G. Dakin, and A. Pomp, "Sleeve gastrectomy for morbid obesity," Obesity Surgery, vol. 17, no. 7, pp. 962-969, 2007.

[14] E. Akkary, "Bariatric surgery evolution from the malabsorptive to the hormonal era," Obesity Surgery, vol. 22, no. 5, pp. 827-831, 2012.

[15] J. Melissas, M. Daskalakis, S. Koukouraki et al., "Sleeve gastrectomy - a "food limiting" operation," Obesity Surgery, vol. 18, no. 10, pp. 1251-1256, 2008.

[16] I. Braghetto, C. Davanzo, O. Korn et al., "Scintigraphic evaluation of gastric emptying in obese patients submitted to sleeve 
gastrectomy compared to normal subjects," Obesity Surgery, vol. 19, no. 11, pp. 1515-1521, 2009.

[17] S. Shah, P. Shah, J. Todkar, M. Gagner, S. Sonar, and S. Solav, "Prospective controlled study of effect of laparoscopic sleeve gastrectomy on small bowel transit time and gastric emptying half-time in morbidly obese patients with type 2 diabetes mellitus," Surgery for Obesity and Related Diseases, vol. 6, no. 2, pp. 152-157, 2010.

[18] T. Baumann, S. Kuesters, J. Grueneberger et al., “Time-resolved MRI after ingestion of liquids reveals motility changes after laparoscopic sleeve gastrectomy-preliminary results," Obesity Surgery, vol. 21, no. 1, pp. 95-101, 2011.

[19] J. Melissas, A. Leventi, I. Klinaki et al., "Alterations of global gastrointestinal motility after sleeve gastrectomy: a prospective study," Annals of Surgery, vol. 258, no. 6, pp. 976-982, 2013.

[20] K. Chandarana and R. L. Batterham, "Metabolic insights from cutting the gut," Nature Medicine, vol. 18, no. 5, pp. 668-669, 2012.

[21] R. Jumpertz, D. S. Le, P. J. Turnbaugh et al., "Energy-balance studies reveal associations between gut microbes, caloric load, and nutrient absorption in humans," The American Journal of Clinical Nutrition, vol. 94, no. 1, pp. 58-65, 2011.

[22] S. H. Duncan, A. Belenguer, G. Holtrop, A. M. Johnstone, H. J. Flint, and G. E. Lobley, "Reduced dietary intake of carbohydrates by obese subjects results in decreased concentrations of butyrate and butyrate-producing bacteria in feces," Applied and Environmental Microbiology, vol. 73, no. 4, pp. 1073-1078, 2007.

[23] J. V. Li, H. Ashrafian, M. Bueter et al., "Metabolic surgery profoundly influences gut microbial-host metabolic crosstalk," Gut, vol. 60, no. 9, pp. 1214-1223, 2011.

[24] H. Zhang, J. K. DiBaise, A. Zuccolo et al., "Human gut microbiota in obesity and after gastric bypass," Proceedings of the National Academy of Sciences of the United States of America, vol. 106, no. 7, pp. 2365-2370, 2009.

[25] J.-P. Furet, L.-C. Kong, J. Tap et al., "Differential adaptation of human gut microbiota to bariatric surgery-induced weight loss: links with metabolic and low-grade inflammation markers," Diabetes, vol. 59, no. 12, pp. 3049-3057, 2010.

[26] N. Runkel, M. Colombo-Benkmann, T. P. Hüttl, H. Tigges, O. Mann, and S. Sauerland, "Bariatric surgery," Deutsches Ärzteblatt, vol. 108, no. 20, pp. 341-346, 2011.

[27] H. Hauner, G. Bucholz, A. Hamann et al., Evidence-Based Guideline: Preventionand Therapy of Obesity, German Obesity Society, German Diabetes Society, German Society for Nutrition, German Society for Nutritional Medicine, 2007, http://www.adipositas-gesellschaft.de/leitlinien.php.

[28] M. A. Kueper, K. M. Kramer, A. Kirschniak, A. Königsrainer, R. Pointner, and F. A. Granderath, "Laparoscopic sleeve gastrectomy: standardized technique of a potential stand-alone bariatric procedure in morbidly obese patients," World Journal of Surgery, vol. 32, no. 7, pp. 1462-1465, 2008.

[29] G. Snyder-Marlow, D. Taylor, and M. J. Lenhard, "Nutrition care for patients undergoing laparoscopic sleeve gastrectomy for weight loss," Journal of the American Dietetic Association, vol. 110, no. 4, pp. 600-607, 2010.

[30] H. R. Gjessing, H. J. Nielsen, G. Mellgren, and O. A. Gudbrandsen, "Energy intake, nutritional status and weight reduction in patients one year after laparoscopic sleeve gastrectomy," SpringerPlus, vol. 2, article 352, 2013.

[31] A. Andreu, V. Moizé, L. Rodríguez, L. Flores, and J. Vidal, "Protein intake, body composition, and protein status following bariatric surgery," Obesity Surgery, vol. 20, no. 11, pp. 1509-1515, 2010.

[32] S. C. Bischoff, A. Damms-Machado, C. Betz et al., "Multicenter evaluation of an interdisciplinary 52-week weight loss program for obesity with regard to body weight, comorbidities and quality of life-a prospective study," International Journal of Obesity, vol. 36, no. 4, pp. 614-624, 2012.

[33] S. Mitra, K. Foerster-Fromme, A. Damms-Machado et al., "Analysis of the intestinal microbiota using SOLiD 16S rRNA gene sequencing and SOLiD shotgun sequencing," BMC Genomics, vol. 14, article S16, 2013.

[34] D. A. Benson, I. Karsch-Mizrachi, D. J. Lipman, J. Ostell, and D. L. Wheeler, "GenBank," Nucleic Acids Research, vol. 33, pp. D34-D38, 2005.

[35] S. F. Altschul, W. Gish, W. Miller, E. W. Myers, and D. J. Lipman, "Basic local alignment search tool," Journal of Molecular Biology, vol. 215, no. 3, pp. 403-410, 1990.

[36] D. H. Huson, S. Mitra, H.-J. Ruscheweyh, N. Weber, and S. C. Schuster, "Integrative analysis of environmental sequences using MEGAN4," Genome Research, vol. 21, no. 9, pp. 1552-1560, 2011.

[37] I. Unterwurzacher, T. Koal, G. K. Bonn, K. M. Weinberger, and S. L. Ramsay, "Rapid sample preparation and simultaneous quantitation of prostaglandins and lipoxygenase derived fatty acid metabolites by liquid chromatography-mass spectrometry from small sample volumes," Clinical Chemistry and Laboratory Medicine, vol. 46, no. 11, pp. 1589-1597, 2008.

[38] M. Rantalainen, O. Cloarec, O. Beckonert et al., "Statistically integrated metabonomic-proteomic studies on a human prostate cancer xenograft model in mice," Journal of Proteome Research, vol. 5, no. 10, pp. 2642-2655, 2006.

[39] I. Nadal, A. Santacruz, A. Marcos et al., "Shifts in clostridia, bacteroides and immunoglobulin-coating fecal bacteria associated with weight loss in obese adolescents," International Journal of Obesity, vol. 33, no. 7, pp. 758-767, 2009.

[40] R. E. Ley, P. J. Turnbaugh, S. Klein, and J. I. Gordon, "Microbial ecology: human gut microbes associated with obesity," Nature, vol. 444, no. 7122, pp. 1022-1023, 2006.

[41] F. B. Langer, M. A. R. Hoda, A. Bohdjalian et al., "Sleeve gastrectomy and gastric banding: effects on plasma ghrelin levels," Obesity Surgery, vol. 15, no. 7, pp. 1024-1029, 2005.

[42] J. V. Li, R. Reshat, Q. Wu et al., "Experimental bariatric surgery in rats generates a cytotoxic chemical environment in the gut contents," Frontiers in Microbiology, vol. 2, article 183, 2011.

[43] D. M. Mutch, J. C. Fuhrmann, D. Rein et al., "Metabolite profiling identifies candidate markers reflecting the clinical adaptations associated with Roux-en-Y gastric bypass surgery," PLoS ONE, vol. 4, no. 11, Article ID e7905, 2009.

[44] E. Munukka, P. Wiklund, S. Pekkala et al., "Women with and without metabolic disorder differ in their gut microbiota composition," Obesity, vol. 20, no. 5, pp. 1082-1087, 2012.

[45] V. Tremaroli and F. Bäckhed, "Functional interactions between the gut microbiota and host metabolism," Nature, vol. 489, no. 7415, pp. 242-249, 2012.

[46] J. A. Parnell and R. A. Reimer, "Weight loss during oligofructose supplementation is associated with decreased ghrelin and increased peptide YY in overweight and obese adults," American Journal of Clinical Nutrition, vol. 89, no. 6, pp. 17511759, 2009.

[47] P. D. Cani, E. Lecourt, E. M. Dewulf et al., "Gut microbiota fermentation of prebiotics increases satietogenic and incretin 
gut peptide production with consequences for appetite sensation and glucose response after a meal," The American Journal of Clinical Nutrition, vol. 90, no. 5, pp. 1236-1243, 2009.

[48] N. M. Delzenne, A. M. Neyrinck, and P. D. Cani, "Modulation of the gut microbiota by nutrients with prebiotic properties: consequences for host health in the context of obesity and metabolic syndrome," Microbial Cell Factories, vol. 10, supplement 1, article S10, 2011.

[49] J. H. Cummings, H. S. Wiggins, D. J. A. Jenkins et al., "Influence of diets high and low in animal fat on bowel habit, gastrointestinal transit time, fecal microflora, bile acid, and fat excretion," Journal of Clinical Investigation, vol. 61, no. 4, pp. 953-963, 1978.

[50] R. Kumar, J. C. Lieske, M. L. Collazo-Clavell et al., "Fat malabsorption and increased intestinal oxalate absorption are common after roux-en-Y gastric bypass surgery," Surgery, vol. 149, no. 5, pp. 654-661, 2011.

[51] B. K. Canales, J. Ellen, S. R. Khan, and M. Hatch, "Steatorrhea and hyperoxaluria occur after gastric bypass surgery in obese rats regardless of dietary fat or oxalate," The Journal of Urology, vol. 190, no. 3, pp. 1102-1109, 2013. 


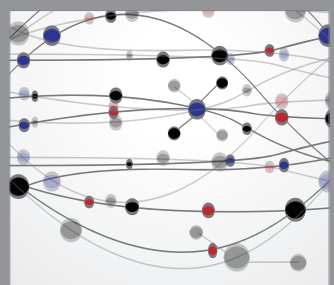

The Scientific World Journal
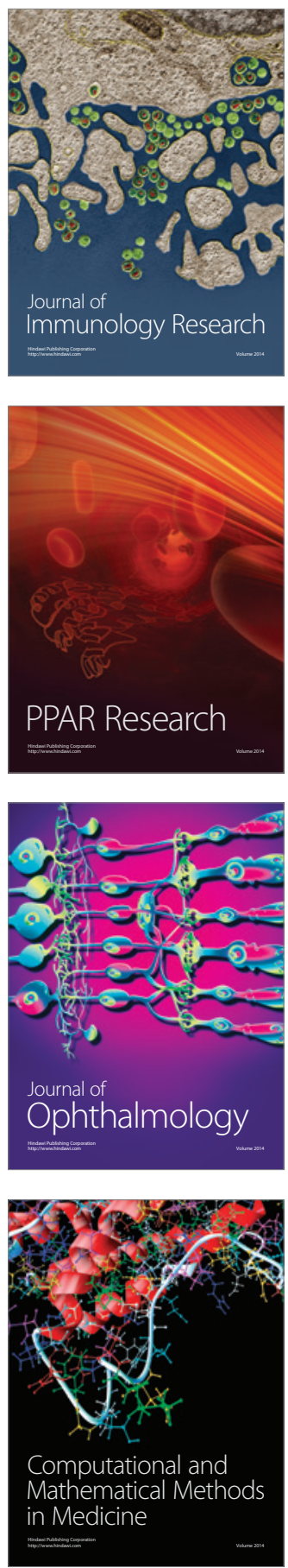

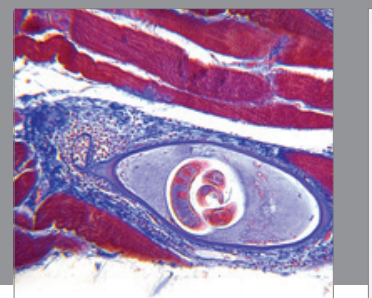

Gastroenterology

Research and Practice
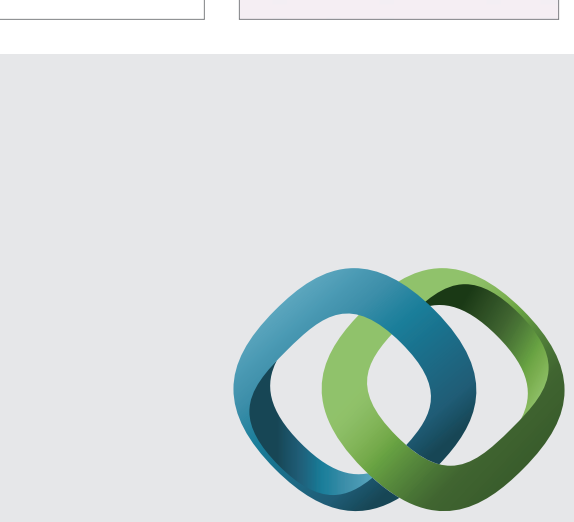

\section{Hindawi}

Submit your manuscripts at

http://www.hindawi.com
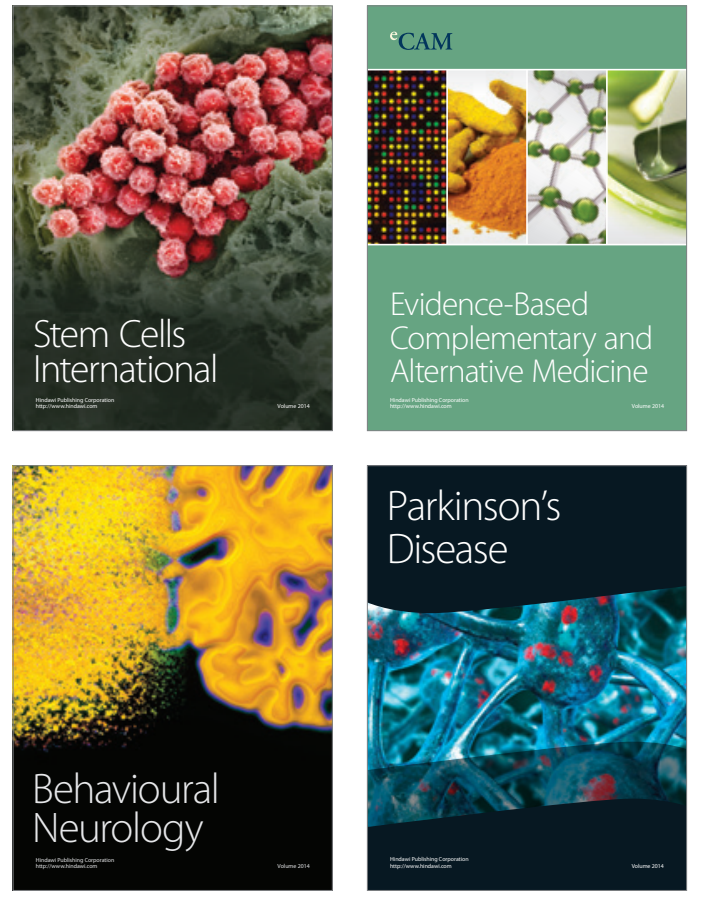
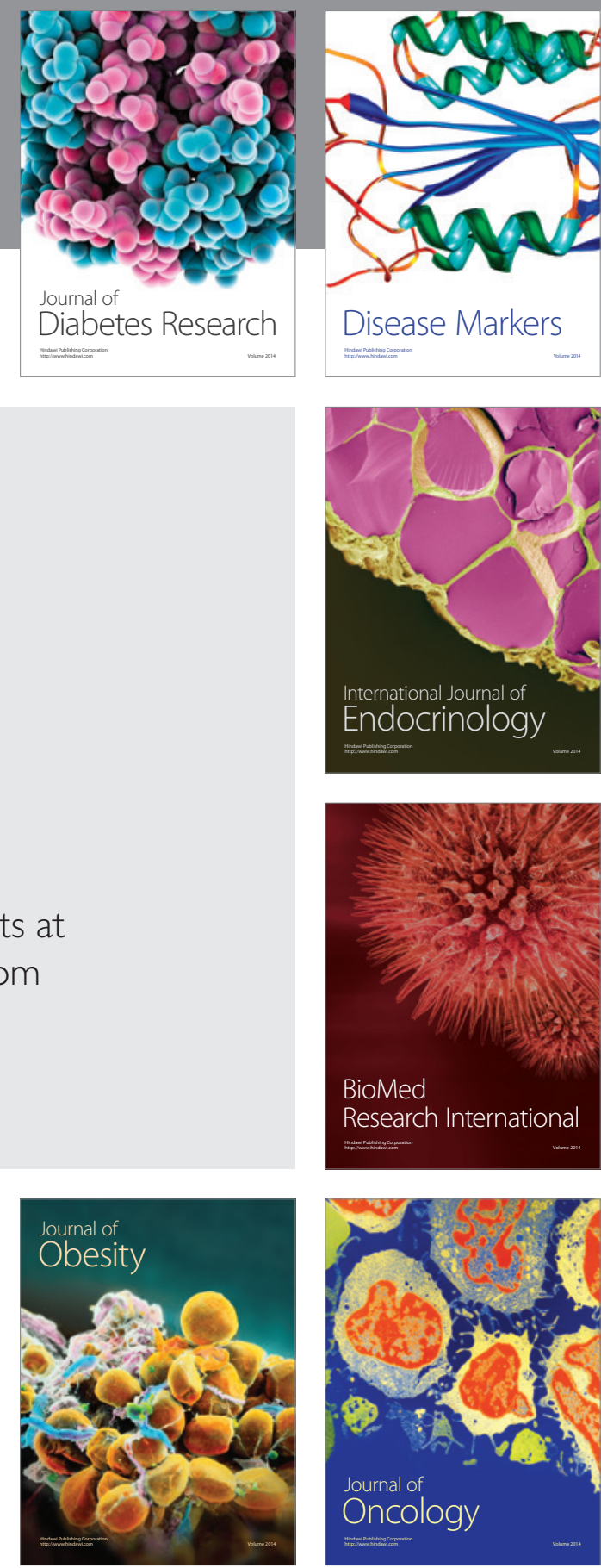

Disease Markers
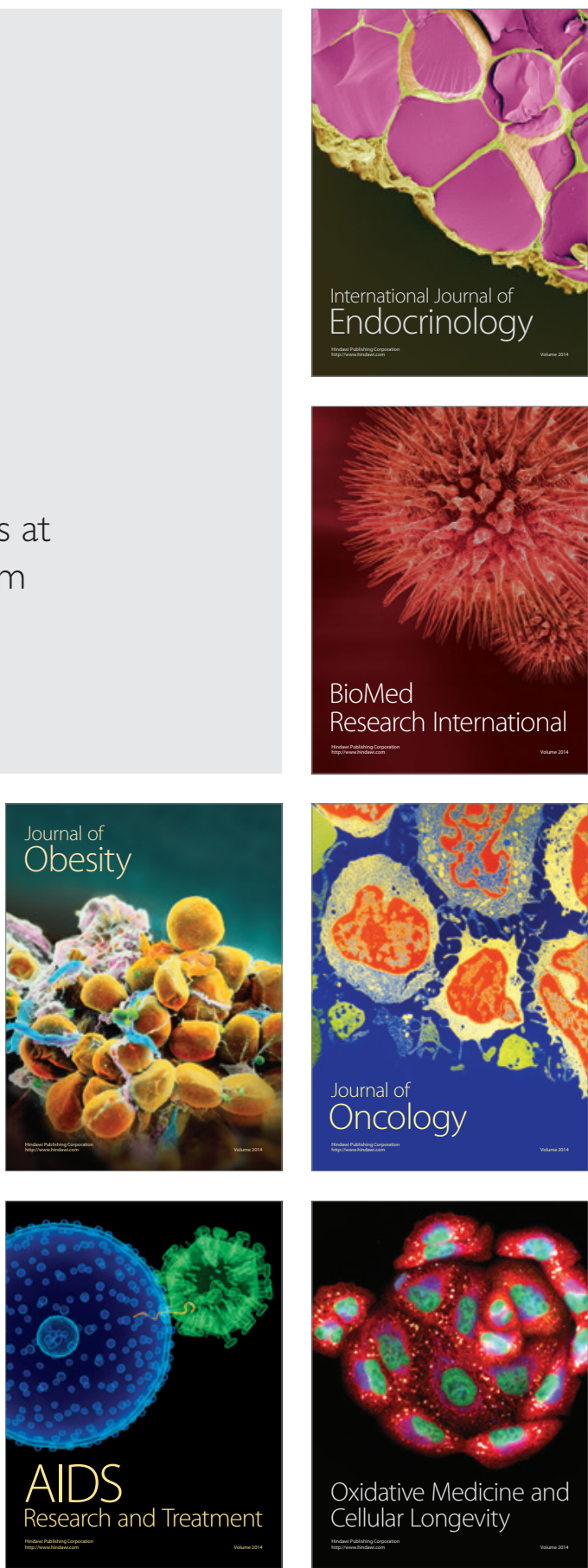